\title{
X-Ray and EUV Observations of CME Eruption Onset
}

\author{
Alphonse C. Sterling ${ }^{1}$ \\ ${ }^{1}$ NASA/MSFC/NSSTC, SD50/Space Science Depart., Huntsville, AL, USA \\ email: alphonse.sterling@nasa.gov
}

\begin{abstract}
.
Why CMEs erupt is a major outstanding puzzle of solar physics. Signatures observable at the earliest stages of eruption onset may hold precious clues about the onset mechanism. We summarize and discuss observations from SOHO/EIT in EUV and from Yohkoh/SXT in soft Xrays of the pre-eruption and eruption phases of three CME expulsions, along with the eruptions' magnetic setting inferred from SOHO/MDI magnetograms. Our events involve clearly-observable filament eruptions and multiple neutral lines, and we use the magnetic settings and motions of the filaments to help infer the geometry and behavior of the associated erupting magnetic fields. Pre-eruption and early-eruption signatures include a relatively slow filament rise prior to eruption, and intensity dimmings and brightenings, both in the immediate neighborhood of the "core" (location of greatest magnetic shear) of the erupting fields and at locations remote from the core. These signatures and their relative timings place observational constraints on eruption mechanisms; our recent work has focused on implications for the so-called "tether cutting" and "breakout" models, but the same observational constraints are applicable to any model.
\end{abstract}

Keywords. Sun: coronal mass ejections (CMEs), Sun: filaments, Sun: flares, Sun: magnetic fields, Sun: UV radiation, Sun: X-rays, gamma rays

\section{Introduction}

Over the past decade it has become clear that a Coronal Mass Ejection (CME) is just one aspect of a general solar magnetic eruption process, which also involves the release of energy in the form of solar flares, and often also involves expulsion of a solar filament. Understanding the solar eruption process is key to eventually being able to predict when eruptions will occur; such understanding will also give us insight into basic solar, stellar, and astrophysical phenomena, many of which are based upon magnetic activity.

Here we report on some of our recent work trying to understand what drives solar eruptions. We have been examining satellite and ground-based data of the onset phase of solar eruptions in an effort to try to understand the eruption trigger mechanism. We primarily consider data from the Soft X-ray Telescope (SXT) on the Yohkoh satellite and from the EUV Imaging Telescope (EIT) on SOHO. We also use magnetograms from SOHO's Michelson Doppler Imager (MDI).

Several models suggest that interactions among coronal magnetic fields are responsible for eruption onset. In order to test these ideas it would be best to directly observe the coronal magnetic field at the start of eruptions, but of course this is not possible. Instead, we have been selecting eruption events which involve observable erupting filaments; such filaments are sometimes visible in absorption in EUV images. We take the motions of the filaments early in the eruption to be a proxy for the pre-eruption and eruption-time evolution of the coronal magnetic fields. In addition to the filaments in the EUV images, images in both EUV and soft X-rays can show prominent intensity brightenings and 
intensity dimmings, and both of these intensity changes can give us further information on the eruption process. In the following, we will present background for two specific models we have been examining, the "tether cutting" and the "breakout" models. We will also discuss "intensity dimmings" and their use as a diagnostic of eruptions. We will then present three examples of eruptions, and consider the implications for these two eruption theories based on observed relative timings of intensity brightenings and dimmings and observed filament motions in the eruption examples.

\section{Two Eruption Theories}

There are several ideas for the cause of solar eruptions; for reviews see, e.g., Forbes (2000), Klimchuk (2001), and Lin et al. (2003). In our recent work we have been using observations to test two specific ideas: the tether cutting model, as developed by Moore \& LaBonte (1980), Sturrock (1989), and Moore et al. (2001); and the "breakout model," put forth by Antiochos (1998) and Antiochos et al. (1999).

Tether cutting holds that the key energy release mechanism for eruptions involves a single highly-sheared magnetic bipole. Reconnection among highly-sheared magnetic fields below a filament (or low in a filament channel) in the core of the bipole initiates and releases the eruption; the reconnection "cuts" (rearranges) magnetic field lines ("tethers") that tie down the core magnetic field, coronal material, and filament (if present), unleashing the sheared core field to erupt.

In contrast to tether cutting, breakout requires a multi-bipolar magnetic field and has the initial reconnection at a neutral point far-removed from the core field that explodes in the eruption. An example is a quadrupole configuration where a highly-sheared "inner" bipole is initially trapped beneath field of an enveloping "outer" bipole. If flux emergence or some other process causes the inner bipole to push upward, then reconnection ("external reconnection") between the inner bipole and outer bipole fields can result in creation of new "side lobe" coronal loops. If the early reconnection between the inner and outer bipoles is slow enough, large stress can build up at the boundary as the slow reconnection progresses. In the model, eventually the reconnection rate increases, and the pent-up inner fields explosively "breakout" through the field of the outer bipole and escape into the heliosphere as a CME. As the inner-bipole field is escaping, tether-cutting-like internal reconnection will occur among its outstretched fields reaching back to the surface, resulting in a standard solar flare.

We can inspect observations for signatures consistent with these models. In particular, breakout predicts activity far from the core during the pre-eruption phase. In contrast, tether cutting requires early activity only in the core region, although this does not preclude concurrent activity far from the core as a byproduct of the core activity.

\section{Intensity Dimmings}

Intensity dimmings are commonly observed in regions associated with eruptions, and they are one tool that we use to assist in checking for eruption signatures consistent with the tether cutting or breakout model. In on-disk coronal images, they are most easily visible in EUV movies, such as those from EIT, and they also can appear in soft X-ray images, such as those from SXT. Some early examples of dimmings are Manoharan et al. (1996), Sterling \& Hudson (1997), Thompson et al. (1998), Thompson et al. (2000), and Gopalswamy \& Hanaoka (1998). Two factors which could be responsible for the dimmings are temperature changes occurring over the duration of the eruption, or mass loss. Howard \& Harrison (2004) and Sterling \& Moore (2004a) present examples of dimmings 
due to temperature changes, but we believe that most of the primary eruption-associated dimmings are due to mass loss rather than temperature change. An argument against temperature-change-induced primary dimmings is that the dimmings frequently occur contemporaneously and cospatially in EIT images (showing $1.5 \mathrm{MK}$ or $2.0 \mathrm{MK}$ plasmas) and SXT images (showing plasmas hotter than 2.0 MK), which argues against the possibility that the plasma is being heated from cooler to hotter coronal temperatures (since this would lead to a brightening in SXT images coinciding with the dimming in EIT). Harra \& Sterling (2001) present more direct evidence for dimmings resulting from mass loss, using spectral data combined with imaging data from the Coronal Diagnostics Spectrometer (CDS) instrument on SOHO.

Frequently, dimmings and brightenings show up best in "difference images" (where an earlier image is subtracted from a later image), although care must be exercised to avoid spurious intensity changes when the difference in time between the two images being subtracted is large, as discussed in Sterling \& Moore (2004a).

\section{Eruption Examples}

Here we present three examples of eruptions. We will summarize our observations of each event, and discuss the possibility that they are initiated by the processes described by the tether cutting or the breakout model. Full details for each case appear in the referenced publications for each respective event.

Broadly speaking, we used two criteria for selecting events for detailed study. First, the events had to include an erupting filament; from the filament motions in the early stages of eruption, we infer properties of the dynamical evolution of the coronal magnetic fields in which the filaments were embedded. Second, we restricted our data sets to events which evolved slowly enough to be resolved by EIT, which has a time cadence of about 12-minutes. Consequently, all of our events are slowly-evolving quiet-region eruptions involving large-scale filaments.

\subsection{Event 1}

This event involves a quiet-region filament eruption of 1999 April 18; Sterling et al. (2001) and Sterling \& Moore (2004a) discuss the event in detail. As we will see in our other examples also, this filament showed a two-stage eruption profile, rising slowly at first and then rapidly at the time of eruption (Fig. 1); in this case the slow-rise phase lasted for about six hours. Post-flare loops and other eruption-related phenomena occurred approximately concurrent with the onset of the fast eruption.

If breakout is responsible for this eruption, then we might expect to see signatures of high-altitude breakout reconnection prior to the onset of the fast-rise phase. Sterling \& Moore (2004a) do indeed observe phenomena consistent with such breakout reconnection occurring during the slow-rise phase. For example, they find subtle brightenings and dimmings in EUV, and brightenings in soft X-rays during the slow rise that are consistent with formation of new side lobe loops in a quadrupolar magnetic geometry, as predicted by breakout. These observations therefore are consistent with early breakout reconnection occurring, although they are not conclusive proof of such reconnection (see Sterling \& Moore (2004a)).

Tether cutting-type of reconnection does occur in this event, in association with a soft $\mathrm{X}$-ray flare and the formation of post-flare loops. The question is: is that reconnection the main agent responsible for triggering the onset of the fast eruption? If so, then we would expect this reconnection to have started prior to the onset of the fast rise. Although the EUV data alone suggest that this may be the case (Sterling et al. (2001)), 


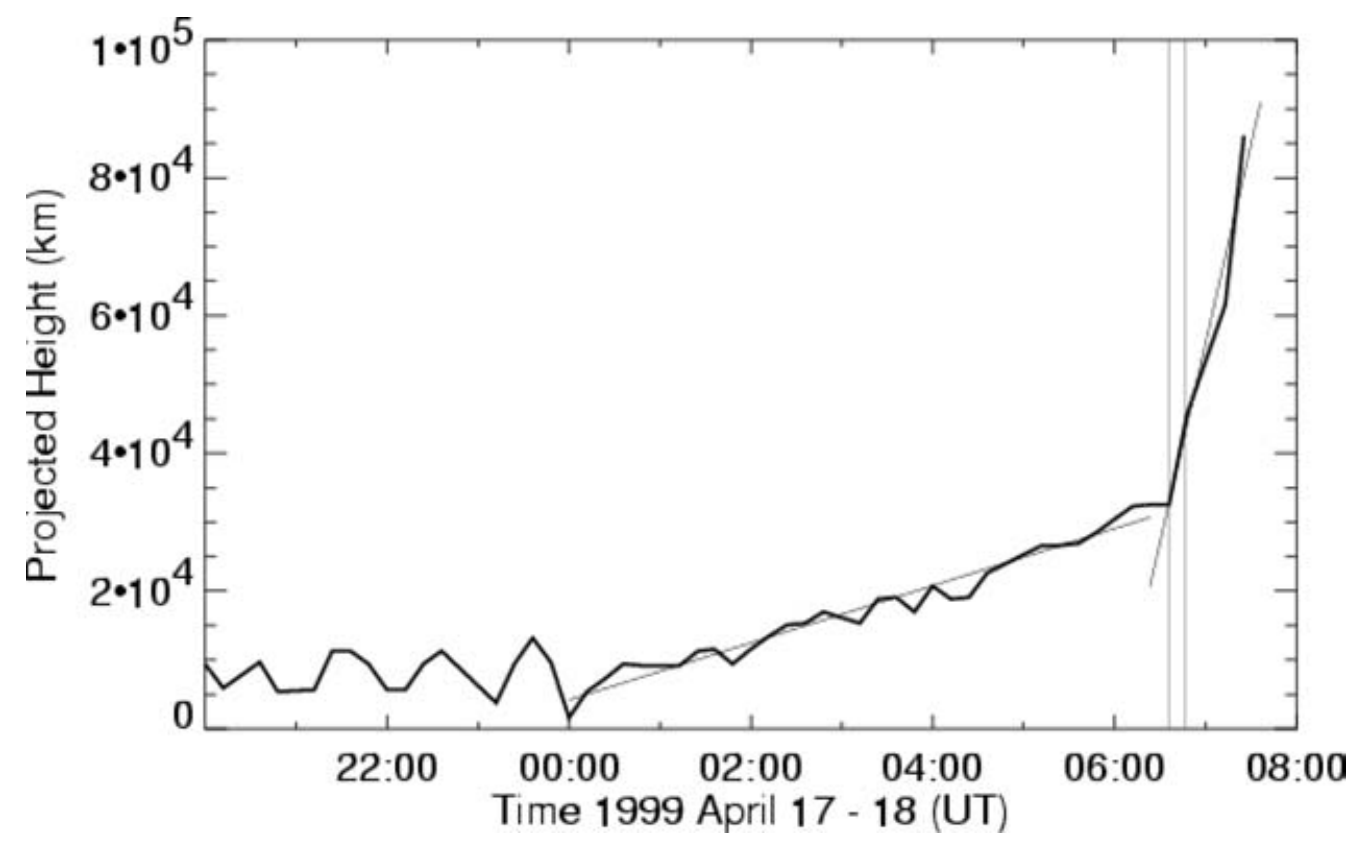

Figure 1. Rise trajectory of an erupting filament as a function of time, where the height is measured projected against the solar disk in EIT $195 \AA$ A images for an event of 1999 April 17-18 (Event 1). A nearly linear slow-phase "pre-eruption" rise between 0 UT and about 6:30 UT is followed by a rapid fast-phase rise; we define the eruption onset as occurring between the times of the two vertical lines. Overplotted on the trajectory are two linear fits, giving line-of-sight velocities of $\sim 1 \mathrm{~km} \mathrm{~s}^{-1}$ and $\sim 15 \mathrm{~km} \mathrm{~s}^{-1}$ for the slow and fast phases, respectively.

Sterling \& Moore (2004a) point out that the soft X-ray emission begins prior to the EUV emission. They were not, however, able to say whether the soft X-ray emission began before or just after the start of the fast rise.

In conclusion, this eruption shows characteristics of both breakout and tether cutting. Supplemental studies, e.g. combining our observations with theoretical predictions, should be able to tell us which, if either, mechanism was actually responsible for the onset of the fast eruption.

\subsection{Event 2}

Our second eruption example occurred on 1999 February $8-9$, and involved a large-scale prominence that erupted from the north polar crown region. It was well observed in the EIT $284 \AA$ (Fe XXV) filter, and by SXT. Sterling \& Moore (2003) give full details of this event.

As in Event 1, this eruption also shows characteristics of a two-phase eruption (Fig. 2), but here the slow-rise phase is not as close to linear as the Event 1 case. This case provides a nice example of concurrent dimmings visible in soft X-rays and EUV, lending further evidence that the dimmings result from mass loss rather than from heating of plasma.

This was a particularly slow eruption, and the associated magnetic fields weak, making it difficult to search for expected pre-eruption breakout signatures. Strong soft X-ray emission did not begin until well after the start of the fast rise of the prominence, and this seems to be inconsistent with the idea that tether cutting reconnection is responsible for the onset of the fast eruption. Sterling \& Moore (2003) show, however, that this is not necessarily the case; tether cutting could have occurred prior to onset of the fast 


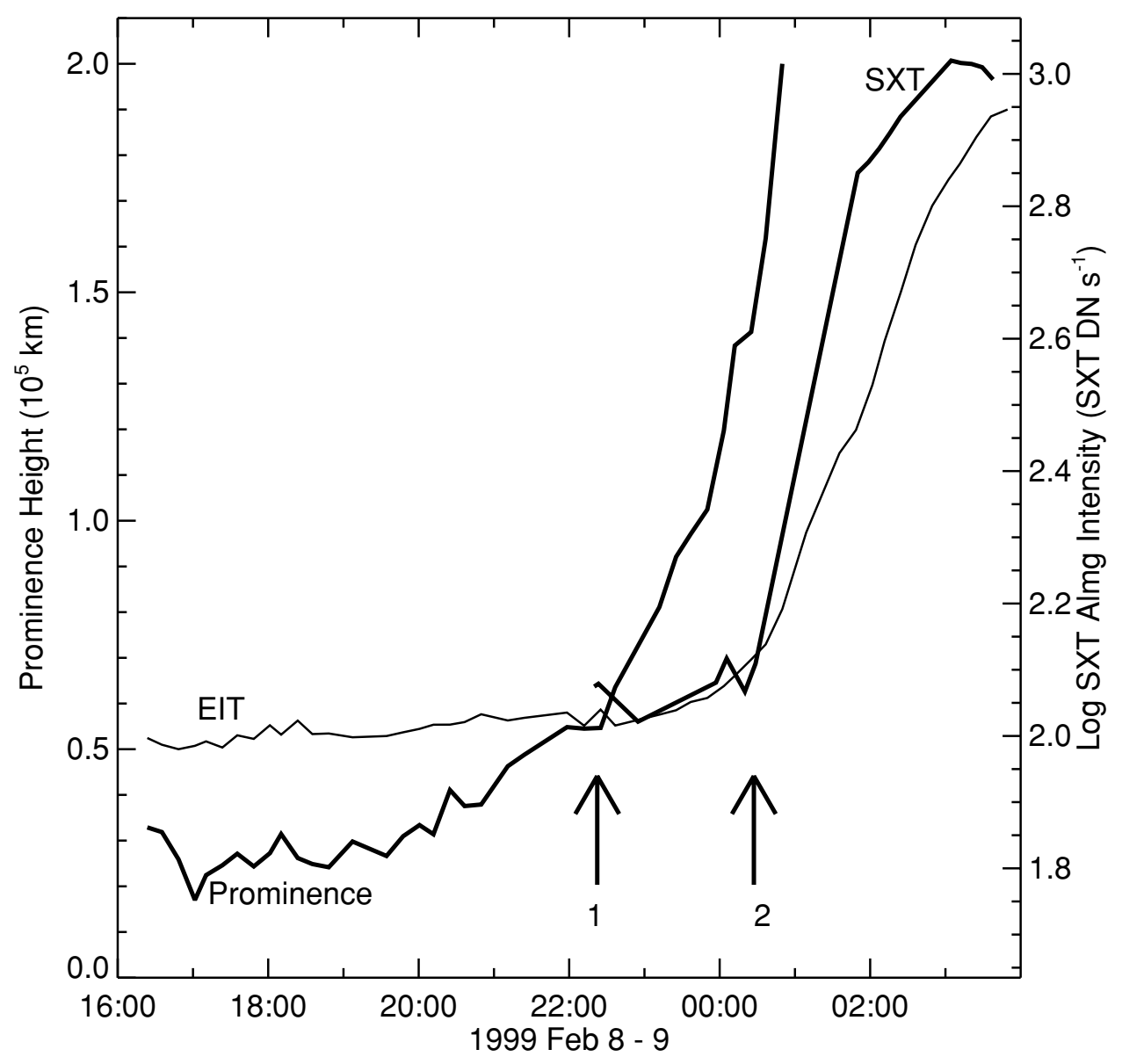

Figure 2. Curve labeled 'prominence' shows the height of the top of the prominence as a function of time for the eruption of $1999 \mathrm{Feb} 8-9$ (Event 2). Curves labeled 'SXT' and 'EIT' respectively show averaged integrated SXT and EIT intensity lightcurves over a spatially-localized region where flare brightenings first occurred (Sterling \& Moore 2003 show the precise location of the region); the EIT lightcurve is plotted with an arbitrary vertical scale. Arrow 1 indicates the time where the prominence trajectory undergoes acceleration, i.e. the start of the transition from the slow-phase to the fast-phase of the eruption. Arrow 2 indicates the time where SXT intensity shows a sharp increase above background level.

eruption, but at such a weak level that soft X-ray emission was lost in the background coronal emission. Therefore, this example does not give us direct evidence for or against either eruption model.

\subsection{Event 3}

This event involved the eruption of a large filament near the solar limb, and was observed in EIT $195 \AA$ images, SXT, and other instruments, as discussed in Sterling \& Moore (2004b). Once again, it showed a two-stage evolution for the filament rise (Fig. 3). In addition to the filament itself, we also follow two features in the corona above the filament, one is a "suspended feature" consisting of a short filament-like patch of cool material, and the other is a bright coronal loop; both moved in conjunction with the rising filament. Sterling \& Moore (2004b) argue that the filament and the suspended feature belonged to the same coronal magnetic cavity, and that the coronal loop was either part of the 


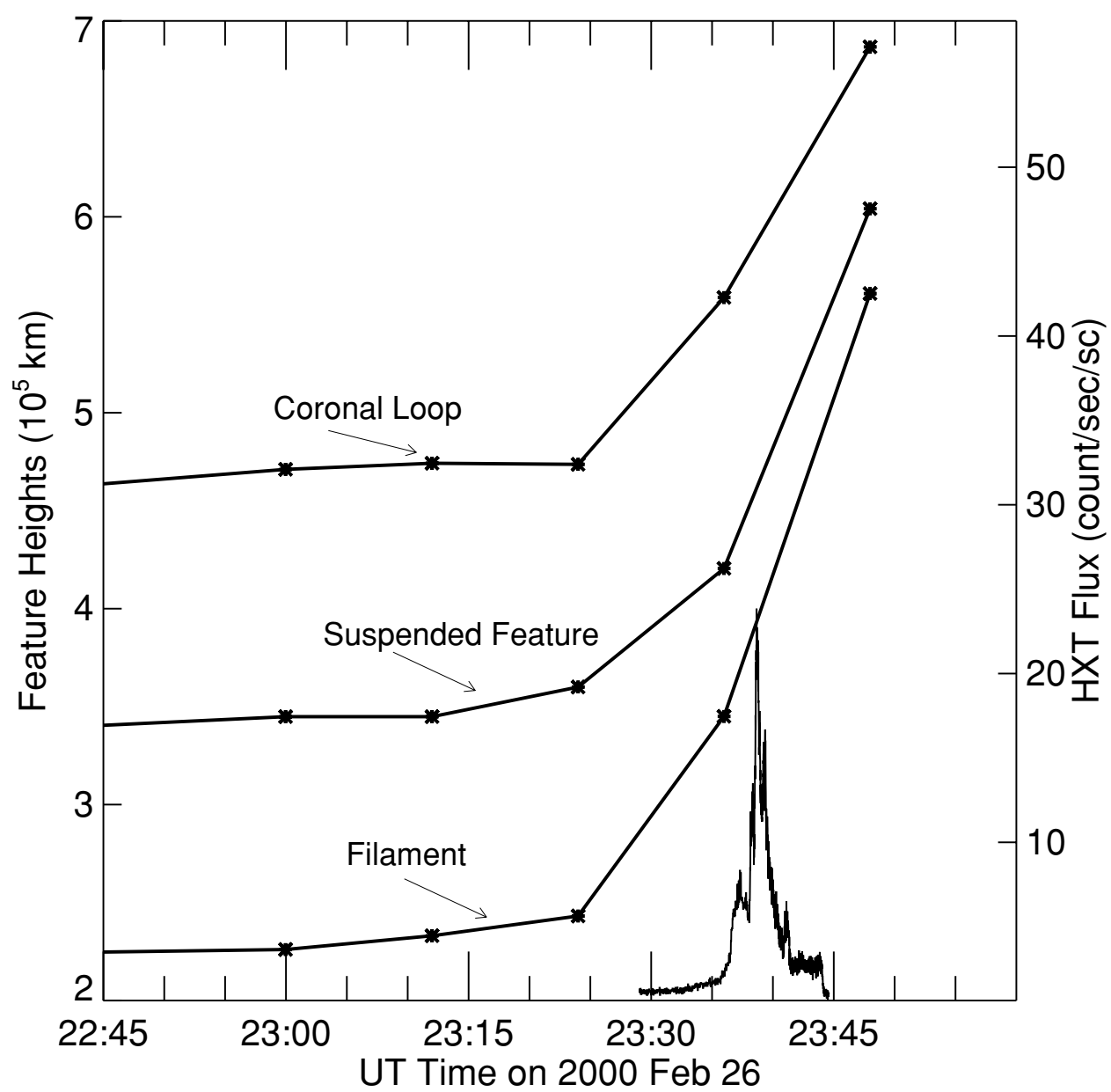

Figure 3. Trajectories of the filament, suspended feature, and coronal loop as functions of time for the eruption of 2000 February 26 (Event 3), measured projected against the disk in EIT images; flux from the Lo channel of Yohkoh's hard X-ray telescope (HXT) is overplotted from 23:29 UT. Slow pre-eruption motion starts near 23:00 UT and fast eruption starts near 23:25 UT.

cavity or arched over the cavity. This example, along with a second, similar example in Sterling \& Moore (2004b), suggest that entire magnetic systems, in this case consisting of the filament, suspended feature, and the larger magnetic cavity in which they resided, underwent a two-stage rise process during eruption.

As with Event 1, this event occurred in a quadrupolar magnetic geometry, and showed evolution consistent with breakout during the pre-eruption slow-rise phase. Also, brightenings occurred in the core region consistent with tether-cutting reconnection. Once again, we need additional information (e.g., combinations of our data with numerical simulations) to tell us whether either of the two models was responsible for triggering the onset of the eruption.

\section{Discussion}

Each of our three filament eruption examples show a slow-rise phase followed by a fast-rise (eruptive) phase, although the change in rise rate is less obvious in the Event 2 
case. This change in rise rate has been observed previously, e.g. Kahler et al. (1988), and similar rise trajectories have been seen in other phenomena (e.g., Ohyama \& Shibata (1997)). For our Events 1 and 3, we find evidence that breakout-type coronal reconnection may be occurring in the respective magnetic systems during the slow-rise phases. For all three cases, tether-cutting-type reconnection occurs, resulting in soft X-ray flares and associated phenomena. With these observations alone we are not able to say conclusively which, if either, of these proposed mechanisms is responsible for triggering the onset of these eruptions. We encourage modelers to combine our observations with detailed numerical simulations mimicking the setup of these specific events; such studies would lend support for or against these (or other) theories. Further detailed morphological and quantitative studies of similar events promise to yield further insights into the eruptiononset process.

\section{Acknowledgements}

This work was supported by funding from NASA's Office of Space Science through the Solar Physics Supporting Research and Technology Program and the Sun-Earth Connection Guest Investigator Program. Yohkoh is a mission of the Institute of Space and Astronautical Sciences (Japan), with participation from the US and $\mathrm{UK}$, and $\mathrm{SOHO}$ is a project of international cooperation between ESA and NASA. The author thanks the IAU Symposium committee for inviting him to speak at this conference.

\section{References}

Antiochos, S. K. 1998, apj 502, L181

Antiochos, S. K., DeVore, C. R., \& Klimchuk, J. A. 1999, apj 510, 485

Forbes, T. G. 2000, JGR 105, 23,153

Gopalswamy, N., \& Hanaoka, Y. 1998, ApJ 498, L179

Harra, L. K. \& Sterling, A. C. 2001, ApJ 561, 215

Howard, T. A., \& Harrison, R. A. 2004, Solar Phys. 219, 315

Hudson, H. S. et al. 1998, Geophys. Res. Lett. 25, 2481

Kahler, S. W. et al. 1998, Solar Phys. 328, 824

Klimchuk, J. A. 2001, in: Space Weather, Geophysical Monograph, 125, 143

Lin, J., Soon, W., \& Baliunas, S. L. 2003, New Astron. Reviews 47, 53

Manoharan, P. K. et al. 1996, ApJ 468, L73

Moore, R. L., \& LaBonte, B. 1980, in: Proc. Symp. on Solar and Interplanetary Dynamics, Reidel, Boston, 207

Moore, R. L., Sterling, A. C., Hudson, H. S., \& Lemen, J. R. 2001, ApJ 552, 833

Ohyama, M., \& Shibata, K. 1997, PASJ 49, 249

Sterling, A. C., \& Hudson, H. S. 1997, ApJ 491, L55

Sterling, A. C., Moore, R. L., \& Thompson, B. J. 2001, ApJ 561, L219

Sterling, A. C., \& Moore, R. L. 2003, ApJ 599, 1418

Sterling, A. C., \& Moore, R. L. 2004a, ApJ 602, 1024

Sterling, A. C., \& Moore, R. L. 2004b, ApJ 613, 1221

Sturrock, P. A. 1989, Solar Phys., 121, 387

Thompson, B. J. et al. 1998, Geophys. Res. Lett. 25, 2465

Thompson, B. J. et al. 2000, Geophys. Res. Lett. 27, 1431 


\section{Discussion}

SCHMiEDER: 1. In the Moore cartoon, there are loops on both sides of the core of the flare which expand. Did you see them in your observations?

2. In your example "events", you say that there is a quadrupolar reconnection. Did you see the brightening of the ribbons corresponding to such a reconnection?

Sterling: 1. Fig. 1 of Moore et al. (2001) shows a cartoon where loops bulge out from both ends of the core; we sometimes refer to these bulging loops as "elbows" to the core field. Prior to eruption we have most often seen these elbow loops in hotter coronal (soft X-ray) images, as in the examples of Moore et al. (2001). But our three events here all occur in quiet regions, where the magnetic fields are not strong enough to result in significant pre-eruption soft X-ray emission. Therefore we do not see these loops before eruption for these cases. For at least the first event, however, we can infer that such loops existed prior to eruption based on double dimming patterns in EUV difference images of that event (see Sterling and Moore 2004a, Fig. 2).

2. Not really, the images are too noisy. We see some evidence of both dimming and brightening at the expected outer ribbon locations, but these are too close to the noise level to be sure; recall that this event is very weak, so such ribbons might be present, but very weak. The inner ribbons correspond to the main flare ribbons, which we do see.

JIE ZHANG: 1. Are EIT waves and dimming different phenomena, or the same phenomena but only different in intensity?

2. The breakout model involves a multipolar region and reconnection at the top. But in terms of the main energy release, do they both involve reconnection in the deep core field? Do they both have the same main energy source?

STERLING: 1. There seems to be at least two phases of wave propagation: (1) A phase where the waves have dimming behind its front, and (2) A phase where the wave continues on after the dimming stops propagating outward. For phase (1), the waves may be the same as the spread of the dimming, but I am not sure about this. For (2), the waves and dimming are almost certainly different. This two-phase wave idea is consistent with the model of Chen \& Shibata (2000) as discussed in Harra \& Sterling (2003).

2. I agree that in breakout and in tether cutting the main energy release is in the core, and you are right in saying that this is an important point. But another important question is "what are the conditions necessary for violent eruption?" The breakout proponents argue that early, slow reconnection far away from the core is essential for violent eruption to start. Tether cutting proponents say that reconnection in the core alone is sufficient for violent eruption.

DelaboudinieRE: What is the role of the filament in the eruption ? - Disappearance of filament coincident with acceleration and change of ionization state of cold matter which is heated at coronal temperature. This is "explosive" and may create the EIT shock(?) waves and dimming. Is a pressure wave pushing the field open after energy has been deposited suddenly from contact between the filament and corona at about 0.5 solar radius altitude?

STERLING: These are good questions that are worthy of future consideration. So far we have only considered the filament to be a passive marker of the coronal magnetic field. 
SHIBATA: 1. You mentioned that the tether-cutting model is fundamentally bipolar, and the breakout model is a multi-polar model. But I think this is not a good classification, because the tether-cutting process can occur in a multi-polar geometry. In other words, an observed multi-polar geometry does not necessarily support the breakout model.

2. In addition to this, your cartoon of event 3 is not the same as the breakout model, although the magnetic field configuration is multi-polar.

3. Your finding that the events tend to show an initial slow rise of a filament followed by a fast rise is very interesting and important. If you normalize the time scale by Alfven time, what will you obtain?

STERLING: 1. Yes, you are correct that multi-polar geometry does not necessarily support breakout; I try to emphasize this point in my papers. Our events 1 and 3 are consistent with breakout, in terms of multi-polar geometry, brightening of side lobes, etc. This does not prove breakout is occurring. Indeed, it could be that some other mechanism is responsible for triggering the eruption in a quadrupole geometry. In that case breakoutlike effects would result as a byproduct of a more fundamental non-breakout mechanism. 2. That event's geometry is different from that of the standard breakout picture, but we argue that event 3 is basically similar to breakout if reconnection at the elevated null point has to occur in order to for the explosive eruption to be triggered. Although we see evidence for reconnection occurring at the elevated null point, we cannot say whether that reconnection is essential for the eruption.

3. Thank you, we are working on doing such a normalization. 